\title{
Short-term Repeat Magnetic Resonance Imaging Scans in Suspected Early Axial Spondyloarthritis Are Clinically Relevant Only in HLA-B27-positive Male Subjects
}

\author{
Raj Sengupta, Helena Marzo-Ortega, Dennis McGonagle, Alison Wadeley, \\ and Alexander N. Bennett, on behalf of the British Society for Spondyloarthritis
}

\begin{abstract}
Objective. Our study investigated the natural history of magnetic resonance imaging (MRI)determined bone marrow edema over a 12-week period in individuals with suspected axial spondyloarthritis.

Methods. There were 109 MRI scans performed on 30 patients who fulfilled the Assessment of Spondyloarthritis international Society inflammatory back pain criteria at baseline and at 4,8 , and 12 weeks.

Results. There were 29 patients who completed the study. Only 4 (14\%) patients changed from MRI-negative to MRI-positive (all HLA-B27-positive, OR 2.74). Three of 7 (43\%) male HLA-B27positive patients, 1 of $8(12.5 \%)$ HLA-B27-positive female patients, and no HLA-B27-negative patients changed from MRI- negative to -positive.

Conclusion. Repeat MRI scans within a 12-week period should be considered in HLA-B27-positive males. (First Release December 1 2017; J Rheumatol 2018;45:202-5; doi:10.3899/jrheum.170171)
\end{abstract}

Key Indexing Terms:

MAGNETIC RESONANCE IMAGING

\section{DIAGNOSIS AXIAL SPONDYLOARTHRITIS}

Sacroiliac joint (SIJ) involvement is a unifying feature in spondyloarthritis (SpA), and radiographic sacroiliitis is a prerequisite for the diagnosis of ankylosing spondylitis (AS). However, radiographic changes may develop slowly, contributing to substantial delays in diagnosis of up to a decade $^{1,2}$. The Assessment of Spondyloarthritis international Society (ASAS) has reclassified SpA into axial (axSpA) and peripheral (pSpA). The ability of magnetic imaging

From the Royal National Hospital for Rheumatic Diseases, Bath; UK National Institute for Health Research (NIHR) Leeds Musculoskeletal Biomedical Research Unit, Leeds Teaching Hospitals Trust; Leeds Institute of Rheumatic and Musculoskeletal Medicine, University of Leeds, Leeds; Culture and Environment College of Liberal Arts, Bath Spa University, Bath; Academic Department of Military Rehabilitation, Defence Medical Rehabilitation Centre, Headley Court, Epsom; University of Bath, Bath, UK. R. Sengupta, MBBS, PGCME, FRCP, Royal National Hospital for Rheumatic Diseases, and University of Bath; H. Marzo-Ortega, LMS, MRCP, PhD, NIHR Leeds Musculoskeletal Biomedical Research Unit, Leeds Teaching Hospitals Trust, and Leeds Institute of Rheumatic and Musculoskeletal Medicine, University of Leeds; D. McGonagle, FRCPI, PhD, NIHR Leeds Musculoskeletal Biomedical Research Unit, Leeds Teaching Hospitals Trust, and Leeds Institute of Rheumatic and Musculoskeletal Medicine, University of Leeds; A. Wadeley, PhD, Culture and Environment College of Liberal Arts, Bath Spa University;

A.N. Bennett, FRCP, PhD, Leeds Institute of Rheumatic and Musculoskeletal Medicine, University of Leeds, and Academic Department of Military Rehabilitation, Defence Medical Rehabilitation Centre.

Address correspondence to Dr. A.N. Bennett, Defence Medical Rehabilitation Centre, Headley Court, Epsom KT186JW, UK. E-mail: alexander.n.bennett@btinternet.com

Accepted for publication September 25, 2017. resonance (MRI) to demonstrate objective evidence of potentially diagnostic inflammatory lesions early in the course of the disease renders it especially useful for the diagnosis of axSpA, particularly when conventional SIJ radiographs are normal or show equivocal changes ${ }^{3,4}$. Further, a positive baseline MRI scan is of prognostic value ${ }^{4,5}$ and may predict response to biologic therapy ${ }^{6}$.

However, the sensitivity of MRI is limited because up to $65 \%$ of subjects with inflammatory back pain (IBP) suspected of having axSpA have a negative SIJ MRI at baseline $^{7}$, and up to $38 \%$ of patients with active AS/axSpA have no inflammatory lesions of the $\mathrm{SIJ}^{6,8}$. AxSpA follows a fluctuating course in early disease and this has been shown both clinically and on MRI ${ }^{9,10}$. Further, the optimal timing of MRI scanning of IBP cases with normal radiographs is poorly defined. One previous study showed that $27 \%$ of HLA-B27-positive patients had a positive repeat MRI scan after 2 years ${ }^{7}$, but clinically, such long waits in symptomatic cases are difficult to justify. Our study evaluates clinically suspected axSpA cases using MRI at 4 timepoints over a 12-week period.

\section{MATERIALS AND METHODS}

Participants were recruited from the Defence Medial Rehabilitation Centre, Headley Court, Epsom, and the Royal National Hospital for Rheumatic Diseases, Bath, UK. Local ethical approval was obtained for our study from the Cornwall and Plymouth Research Ethics Committee (reference number 11/SW/0038) and all patients provided informed written consent. Inclusion

Personal non-commercial use only. The Journal of Rheumatology Copyright $($ C 2018. All rights reserved. 
criteria were IBP as defined by the ASAS criteria ${ }^{11}$ and normal SIJ on anteroposterior pelvis radiographs (scored centrally). Because it is unclear whether nonsteroidal antiinflammatory drugs (NSAID) might affect MRI bone marrow edema (BME), participants were asked to either discontinue all NSAID use or to continue taking a regular dose throughout the study duration to ensure consistency between scans. Additional simple analgesia was permitted if required. Recruited subjects had a total of 4 MRI scans using a predefined protocol at baseline and at 4, 8, and 12 weeks. The protocol included sagittal T1 and short-tau inversion recovery of the cervicothoracic spine, thoracolumbar spine, and coronal oblique of the SIJ. At each visit, patient-reported outcome measures (Bath indices), medication history, and C-reactive protein (CRP) were recorded.

MRI scans. MRI scans were scored according to the Leeds Scoring system $^{8,10}$. In addition, all images were assessed for ASAS definitions of a positive scan of the SIJ ${ }^{12}$ and spine ${ }^{13}$, thereafter referred to as "positive" or "negative" depending on whether definitions were fulfilled, respectively. In the SIJ, although the readers' focus was on BME lesions, if BME lesions were equivocal and not necessarily highly suggestive of axSpA, the additional presence of structural lesions (erosion, sclerosis, fatty deposition, or fusion) could be used to influence the decision as per the updated ASAS definition ${ }^{14}$. Experienced readers (HMO and DMG) scored the scans and radiographs, and were blinded for any clinical data and timepoints. Any disagreement or borderline/equivocal scans were settled by consensus. Radiographs were scored according to the modified New York Criteria $(\mathrm{mNYC})^{1}$. Intrareader reliability for ASAS MRI-positive definitions at the SIJ and spine were considered excellent with $\kappa(\mathrm{SE})^{15}=0.80(0.19), \mathrm{p}=0.01$, and $1.00(0.00), \mathrm{p}<0.0001$, respectively. Reliability for $\mathrm{mNYC}$ classification of radiographs had $100 \%$ agreement $(\mathrm{SE})=1.00(0.00), \mathrm{p}<0.0001$ (absolute agreement).

\section{RESULTS}

There were 30 participants recruited (Table 1), of whom 29 completed the study, 25 (86\%) attended all 4 visits, 2 patients attended 3 visits, and 1 patient attended 2 visits. A total of

Table 1. Baseline characteristics. Values are n (\%) unless otherwise specified.

\begin{tabular}{lc}
\hline Characteristics, $\mathrm{n}=29$ & Values \\
\hline HLA-B27-positive & $21(72)$ \\
Male & $19(66)$ \\
Symptom duration, yrs, mean \pm SD & $5 \pm 2.1$ \\
Fulfilled ASAS clinical criteria & $17(59)$ \\
Fulfilled ASAS imaging criteria & $12(41)$ \\
SpA features, $\geq 2$ & $25(86)$ \\
Uveitis & $3(10)$ \\
Psoriasis & $9(31)$ \\
IBD & 0 \\
Regular NSAID & $13(45)$ \\
Baseline outcome measures, mean \pm SD & \\
$\quad$ BASDAI & $4.8 \pm 0.7$ \\
Back pain score & $5.7 \pm 1$ \\
BASFI & $3.5 \pm 0.7$ \\
ASDAS & $2.5 \pm 0.3$ \\
$\quad$ CRP & $2.7 \pm 1.7$ \\
Raised CRP & $8(29)$
\end{tabular}

ASAS: Assessment of Spondyloarthritis international Society; ASDAS: Ankylosing Spondylitis Disease Activity Score; BASDAI: Bath Ankylosing Spondylitis Disease Activity Index; BASFI: Bath Ankylosing Spondylitis Functional Index; CRP: C-reactive protein; IBD: inflammatory bowel disease; NSAID: nonsteroidal antiinflammatory drugs; SpA: spondyloarthritis.
109 MRI scans were performed. As well as meeting the inclusion criteria, all patients had $\geq 1$ clinical SpA feature (Table 2) and $25(86 \%)$ had $\geq 2$. There were 13 patients (45\%) who took regular NSAID throughout the study. All patients were assessed by ANB and RS. Nonradiographic axSpA was diagnosed in $79 \%$ and mechanical back pain in $21 \%$. On application of ASAS classification, 26\% met the imaging arm, 39\% met the clinical arm, and 35\% met both arms.

ASAS status. There were 17 participants (59\%) who met the ASAS clinical criteria for axSpA prior to any imaging. Eleven (38\%) met ASAS imaging arm classification criteria during the study, and a further $3(10 \%)$ had a positive MRI of the spine but normal SIJ (HLA-B27-positive and $\geq 1$ clinical feature).

There were 15 (52\%) participants who were ASAS MRI-negative (spine and SIJ) at baseline and remained so during the entire study, and 10 (34\%) patients were either spine- and/or SIJ-positive for the duration of the study. A total of $4(14 \%)$ patients changed from ASAS axSpA MRI-negative to -positive (spine, $\mathrm{n}=3$ or SIJ, $\mathrm{n}=1$ ) during the study, which influenced their final diagnosis (Figure 1).

CRP status and change. Of the 28 patients who had blood test results available, CRP levels were normal in 25 subjects (89\%) at baseline: 20 remained normal throughout and 5 patients' CRP became elevated above the upper limit of normal during the study. Three subjects had a raised CRP at baseline: 1 remained raised throughout study and 2 normalized. There was no relationship between CRP change and MRI scans becoming positive at any time during the study.

HLA-B27 status and sex. All 4 patients whose MRI changed from negative to positive were HLA-B27-positive. Comparing patients who were HLA-B27-positive with those who were HLA-B27-negative, the OR for their ASAS MRI status changing from negative to positive was 2.74 .

Three of the 4 patients $(75 \%)$ whose MRI changed from negative to positive were male. Of the 10 patients whose MRI scans were positive throughout, 9 were male $(90 \%)$. Of the 15 patients who were HLA-B27-positive with negative baseline scans, 4 (27\%) developed positive scans during the 12-week study period. Three of 7 (43\%) HLA-B27-positive male patients, compared to 1 out of $8(12.5 \%)$ HLA-B27positive female patients and no HLA-B27-negative patients, changed from ASAS MRI definition-negative to positive during the study.

\section{DISCUSSION}

A positive MRI aids diagnosis in clinically suspected axSpA; however, MRI may be negative or inconclusive in a significant proportion of these patients ${ }^{7}$. Our study tested the hypothesis that repeat MRI scanning in suspected but undiagnosed axSpA over 12 weeks may increase the diagnostic yield. In the studied cohort of IBP patients with typical

$$
\text { Personal non-commercial use only. The Journal of Rheumatology Copyright }(\subset) 2018 \text {. All rights reserved. }
$$


Table 2. Distribution of SpA features by HLA-B27 status in study population.

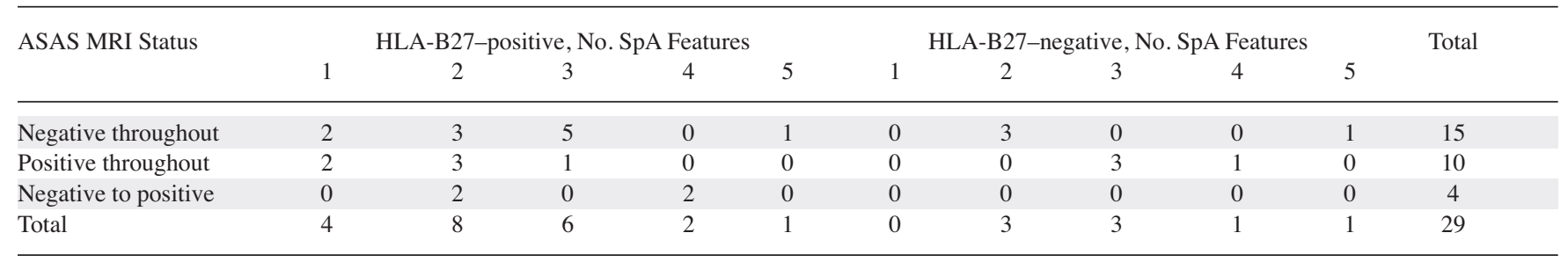

ASAS: Assessment of Spondyloarthritis international Society; MRI: magnetic resonance imaging; SpA: spondyloarthritis.

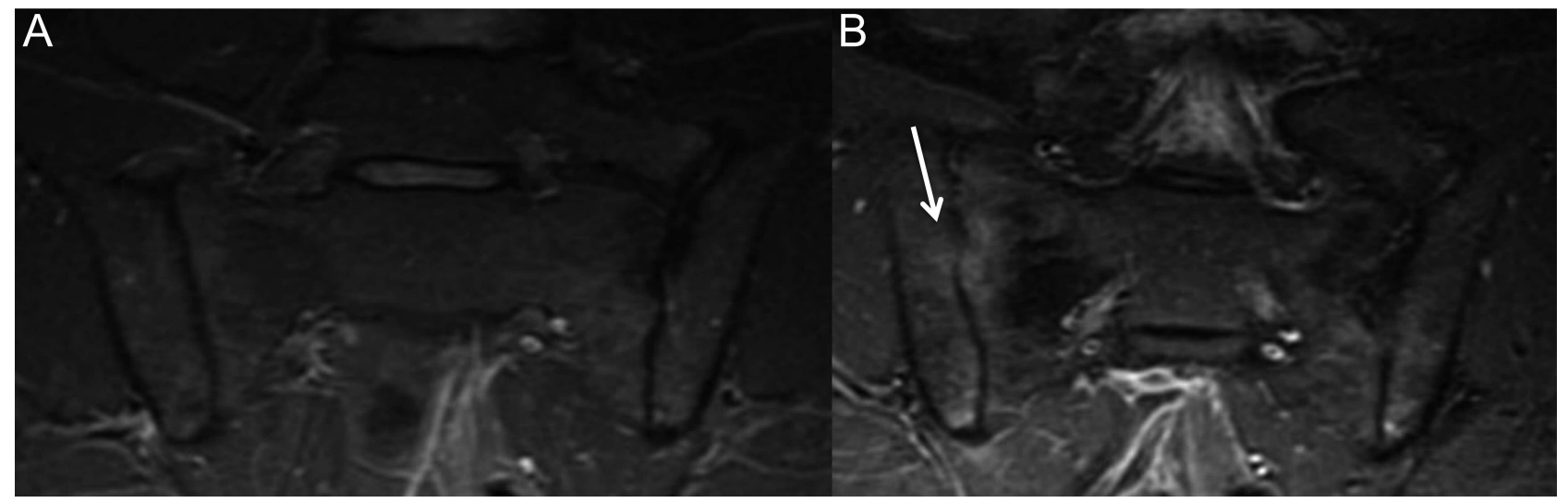

Figure 1. MRI changes in 1 patient between (A) the negative scan at baseline; and (B) the development of active inflammatory sacroiliitis (arrow) in the right SIJ (present on $>1$ slice) at Week 4, which resulted in a change from ASAS SIJ definition "negative" to "positive". ASAS: Assessment of Spondyloarthritis international Society; MRI: magnetic resonance imaging; SIJ: sacroiliac joint.

axSpA features (HLA-B27-positive $=72 \%, \geq 2$ SpA features $=86 \%$, only $14 \%$ of patients changed their ASAS MRI definition from negative to positive over the 12-week period. While the yield of repeating an MRI scan in patients with IBP and a normal baseline scan is low, there may be benefit in repeating the scan within 12 weeks, especially in HLA-B27positive males, because $43 \%$ of these patients changed from negative MRI to positive during the study period. Neither baseline CRP nor presence of other SpA features (dactylitis, arthritis, enthesitis) predicted change in MRI status.

The majority of our cohort (59\%) met ASAS clinical criteria at baseline. There were 52\% who were ASAS imaging-negative at baseline and remained so throughout the study. Overall, in our 12-week study, there was little change in MRI inflammatory lesions.

The natural variation of inflammatory MRI lesions of BME in axSpA remains poorly defined. From an SIJ perspective, 1 study showed that $73.5 \%$ of patients who had SIJ BME at baseline had persistent lesions 1 year later ${ }^{10}$, even in the absence of clinical symptoms. In the spine, $31 \%$ of vertebral corners showed persistence of inflammatory lesions at a 2 -year timepoint ${ }^{16}$. These observations, together with the findings from our study, suggest that once an inflammatory lesion develops, there is likely to be little change in the spine and in SIJ lesions within the first year unless biologic therapies are introduced. However, our study supports the findings of van Onna, et $a l^{7}$ that if the baseline scan is negative, then the proportion changing to a positive scan at followup is higher in HLA-B27-positive patients (27\%) compared to patients who are HLA-B27-negative (0\%).

The strengths of our study include the realistic clinical time frames for potential followup scans and the fact that the study was specifically designed to investigate the usefulness of repeat scans within a 12-week window, which has not previously been performed, to our knowledge. Other strong aspects include the minimal absence of followup MRI scans in the cohort as well as data on NSAID use collected on all patients throughout the study. The main limitations of our study were the lack of asymptomatic and/or mechanical back pain control arms.

Our study evaluated the natural history of axSpA-related inflammatory lesions on MRI and the role of repeat MRI scanning within a 12 -week period in clinically suspected early axSpA. In a symptomatic patient with suspected axSpA, where baseline MRI scanning is normal, a repeat MRI scan may be of diagnostic utility in HLA-B27-positive males but not in females or HLA-B27-negative subjects. These results are highly relevant to aid in clinical decision making and to add to the body of evidence on MRI use in axSpA diagnosis. 


\section{REFERENCES}

1. van der Linden S, Valkenburg HA, Cats A. Evaluation of diagnostic criteria for ankylosing spondylitis. A proposal for modification of the New York criteria. Arthritis Rheum 1984;27:361-8.

2. Mau W, Zeidler H, Mau R, Majewski A, Freyschmidt J, Stangel W, et al. Clinical features and prognosis of patients with possible ankylosing spondylitis. Results of a 10-year follow-up. J Rheumatol 1988;15:1109-14.

3. Heuft-Dorenbosch L, Landewe R, Weijers R, Wanders A, Houben $\mathrm{H}$, van der Linden $\mathrm{S}$, et al. Combining information obtained from magnetic resonance imaging and conventional radiographs to detect sacroiliitis in patients with recent onset inflammatory back pain. Ann Rheum Dis 2006;65:804-8.

4. Bennett AN, McGonagle D, O'Connor P, Hensor EM, Sivera F, Coates LC, et al. Severity of baseline magnetic resonance imaging-evident sacroiliitis and HLA-B27 status in early inflammatory back pain predict radiographically evident ankylosing spondylitis at eight years. Arthritis Rheum 2008;58:3413-8.

5. Maksymowych WP, Chiowchanwisawakit P, Clare T, Pedersen SJ, Ostergaard M, Lambert RG. Inflammatory lesions of the spine on magnetic resonance imaging predict the development of new syndesmophytes in ankylosing spondylitis: evidence of a relationship between inflammation and new bone formation. Arthritis Rheum 2009;60:93-102.

6. Rudwaleit M, Schwarzlose S, Hilgert ES, Listing J, Braun J, Sieper $\mathrm{J}$. MRI in predicting a major clinical response to anti-tumour necrosis factor treatment in ankylosing spondylitis. Ann Rheum Dis 2008;67:1276-81.

7. van Onna M, Jurik AG, van der Heijde D, van Tubergen A, Heuft-Dorenbosch L, Landewe R. HLA-B27 and gender independently determine the likelihood of a positive MRI of the sacroiliac joints in patients with early inflammatory back pain: a 2-year MRI follow-up study. Ann Rheum Dis 2011;70:1981-5.

8. Bennett AN, Rehman A, Hensor EM, Marzo-Ortega H, Emery P, McGonagle D. Evaluation of the diagnostic utility of spinal magnetic resonance imaging in axial spondylarthritis. Arthritis Rheum 2009;60:1331-41.
9. Stone MA, Pomeroy E, Keat A, Sengupta R, Hickey S, Dieppe P, et al. Assessment of the impact of flares in ankylosing spondylitis disease activity using the Flare Illustration. Rheumatology 2008;47:1213-8.

10. Marzo-Ortega H, McGonagle D, O’Connor P, Hensor EM, Bennett AN, Green MJ, et al. Baseline and 1-year magnetic resonance imaging of the sacroiliac joint and lumbar spine in very early inflammatory back pain. Relationship between symptoms, HLA-B27 and disease extent and persistence. Ann Rheum Dis 2009;68:1721-7.

11. Sieper J, van der Heijde D, Landewe R, Brandt J, Burgos-Vagas R, Collantes-Estevez E, et al. New criteria for inflammatory back pain in patients with chronic back pain: a real patient exercise by experts from the Assessment of SpondyloArthritis international Society (ASAS). Ann Rheum Dis 2009;68:784-8.

12. Rudwaleit M, Jurik AG, Hermann KG, Landewe R, van der Heijde $\mathrm{D}$, Baraliakos $\mathrm{X}$, et al. Defining active sacroiliitis on magnetic resonance imaging (MRI) for classification of axial spondyloarthritis: a consensual approach by the ASAS/OMERACT MRI group. Ann Rheum Dis 2009;68:1520-7.

13. Hermann KG, Baraliakos X, van der Heijde DM, Jurik AG, Landewé R, Marzo-Ortega H, et al. Descriptions of spinal MRI lesions and definition of a positive MRI of the spine in axial spondyloarthritis: a consensual approach by the ASAS/OMERACT MRI study group. Ann Rheum Dis 2012;71:1278-88.

14. Lambert RG, Bakker PA, van der Heijde D, Weber U, Rudwaleit M, Hermann KG, et al. Defining active sacroiliitis on MRI for classification of axial spondyloarthritis: update by the ASAS MRI working group. Ann Rheum Dis 2016;75:1958-63.

15. Cohen J. Weighted kappa: nominal scale agreement with provision for scaled disagreement or partial credit. Psychol Bull 1968; 70:213-20.

16. Baraliakos X, Listing J, Rudwaleit M, Sieper J, Braun J. The relationship between inflammation and new bone formation in patients with ankylosing spondylitis. Arthritis Res Therapy 2008;10:R104. 